\title{
Feet distance and static postural balance: Implication on the role of natural stance
}

\author{
Ji-Won Kim a , Yuri Kwon ${ }^{\mathrm{a}}$, Hyung-Min Jeon ${ }^{\mathrm{a}}$, Min-Jung Bang ${ }^{\mathrm{a}}$, Jae-Hoon Jun ${ }^{\mathrm{a}, \mathrm{b}}$, Gwang- \\ Moon Eom ${ }^{\mathrm{a}, \mathrm{b},{ }^{*}}$ and Do-Hyung Lim ${ }^{\mathrm{c}}$ \\ ${ }^{a}$ The School of Biomedical Engineering, Konkuk University, Chungnju, 380-701, Korea \\ ${ }^{b}$ Research Institute of Biomedical Engineering, Konkuk University, Chungnju, 380-701, Korea \\ ${ }^{c}$ Department of Mechanical Engineering, Sejong University, Seoul, 143-747, Korea
}

\begin{abstract}
The purpose of this study was to investigate 1) the effect of feet distance on static postural balance and 2) the location of natural feet distance and its possible role in the relationship of feet distance and postural balance. Static balance tests were performed on a force platform for $100 \mathrm{~s}$ with six different feet distances $(0,5,10,15,20,25 \mathrm{~cm})$. Measures of postural balance included mean amplitude of horizontal ground reaction force (GRF) as well as the mean distance and velocity of the center of pressure (COP). All measures were discomposed into anterioposterior and mediolateral directions. ANOVA and post-hoc comparison were performed for all measures with feet distance as an independent factor. Also measured was the feet distance at the natural stance preferred by each subject. All measures significantly varied with feet distance $(p<0.001)$. Mean distance of COP showed monotonic decrease with feet distance. Mean amplitude of horizontal GRF as well as mean velocity of COP showed U-shaped pattern (decrease followed by increase) with the minimum at the feet distance of $15 \mathrm{~cm}$ or $20 \mathrm{~cm}$, near which the natural feet distance of 16.5 (SD 3.8) $\mathrm{cm}$ was located. COP is regarded to be an approximation of the center of mass (hence the resultant performance of postural control) in an inverted pendulum model with the horizontal GRF ignored. On the other hand, horizontal GRF is the direct cause of horizontal acceleration of a center of mass. The present result on horizontal GRF shows that the effort of postural control is minimized around the feet distance of natural standing and implies why the natural stance is preferred.
\end{abstract}

Keywords: Stance width, postural stability, preferred stance, center of pressure, ground reaction force

\section{Introduction}

Postural balance measured by static posturography was suggested as an important risk factor of falls but those by dynamic posturography were not predictive of falls probably from the ceiling effect [1]. Inconsistent stance conditions (feet together, narrow and natural stance) used in the literature which investigated static postural stability resulted in inconsistencies in the effects of gender, age and other factors $[2,3]$. Obviously, the stance condition must be standardized for the comparison of the results, based on concrete understanding of the effect of feet distance on static postural stability.

The effect of a stance condition on the dynamic postural stability was recently investigated using unexpected platform disturbances [4-6] and also using centripetal accelerations on a rotating platform

${ }^{*}$ Corresponding author: Gwang-Moon Eom, The School of Biomedical Engineering, Konkuk University, Chungnju, 380701, Korea. Tel.: +82-43-840-3766; Fax: +82-43-851-0620; E-mail: gmeom@kku.ac.kr.

0959-2989/14/\$27.50 @ 2014 - IOS Press and the authors. 
$[7,8]$. However, there have been only few investigations on the influence of feet distance on the static postural stability $[9,10]$.

Kirby et al. [9] compared four feet distances in ten subjects. They showed that total sway path of the center of pressure (COP) in the mediolateral direction is greater for feet-together stance than for the other feet distances. They found no difference in the sway path in the anteroposterior direction. Afterwards, Day et al. [10] compared 5 feet distances in 35 subjects. Their measures included the variations in COP and body markers and showed that the measures decreased with feet distance in both anteroposterior and mediolateral directions.

The motivation of this study was a question about the role of natural stance in the middle of various feet distances. The question was, 'can the preference of natural stance be explained in terms of the postural stability? To answer for the question, we need a measure for the causal effort of postural control as well as the resultant performance of postural control.

It was suggested by the inverted pendulum model that the gross movement pattern of center of mass (COM) of a whole body is similar to that of COP, i.e., both signals are virtually in phase and the dynamic range of COP is slightly greater than that of COM [11]. It was also assumed in the model that ankle joint moment move the COP thereby regulate COM, so that the reversals of COM coincide with an overshoot of the COP signal. In this respect, variation of COP has been regarded as the measure of postural balance performance in that it can approximate the variation in COM (postural sway). According to above, it is apparent that the COP variation is the resultant measure of postural balance.

On the other hand, horizontal ground reaction force (GRF) is a direct cause of horizontal acceleration of COM [12], i.e., it reflects the amount of muscular effort in generating horizontal acceleration to maintain balance. GRF measures were shown to have better reliability than those of COP measures [13], and were used to investigate the postural steadiness in different age groups [14].

The hypothesis of this study was that the natural feet distance may be one that can minimize the horizontal GRF (the postural control activity) and this may not be shown in COP variations (the postural control result). To test the hypothesis, we evaluated horizontal GRF as well as COP at six feet distances and also investigated the feet distance of natural stance.

\section{Methods}

\subsection{Subjects}

Forty healthy young subjects (20 male and 20 females) aged 22.5 (1.8) Yrs participated in this study with informed consent. Subjects with diabetes were also excluded because diabetes is reported to be associated with postural stability [15] Subjects who majored in sports were also excluded, because they were regarded as deviant from the normal population. All subjects gave informed consent

\subsection{Measurements}

All subjects performed static balance tests on a force platform (OR6-7, AMTI, Watertown, MA) under 6 different feet distances. Feet distance was defined as the distance between the centers of an inside border of each foot. Selected feet distance ranged from feet together stance $(0 \mathrm{~cm})$ to a wide stance $(25 \mathrm{~cm})$, in $5 \mathrm{~cm}$ intervals.

Each subject stood with arms at the sides, and looked straight at a visual reference (black-colored circle with a diameter of $10 \mathrm{~cm}$ at eye level on the wall at a distance of $2.5 \mathrm{~m}$ ). The forces and mo- 
ments were measured by strain gages attached to load cells near the four corners of the force platform. COP and GRF were derived from the forces and moments at the load cells. They were collected by EvaRT software (Version 5.0.4, Motion Analysis Corporation) for100s with the sampling of $120 \mathrm{~Hz}$. The balance tests were performed three times. All subjects had a seated rest about five minutes between the trials.

Also measured was the feet distance at the natural stance preferred by each subject. It was measured three times per subject.

\subsection{Analysis}

A $4^{\text {th }}$ - order zero phase Butterworth lowpass digital filter with a $10 \mathrm{~Hz}$ cutoff frequency was used for the filtering of COP and GRF data. $10 \mathrm{~Hz}$ was selected as it was proposed as a standard cutoff frequency of force plate measures from the sensitivity analysis by Schmid et al. [16]. First and last $5 \mathrm{~s}$ of filtered signal was excluded in subsequent analysis (hence $90 \mathrm{~s}$ of data were used), because they may contain transient response affected by filtering.

Outcome measures were derived from the COP and GRF in anteroposterior and mediolateral directions. As COP outcome measures, mean distance (MD) and mean velocity (MV) of COP were calculated. MD was defined as the average distance of instantaneous COP from COP0 (the mean COP) as in Eq. (1). MV was defined as the total length of sway path divided by the total sway time, $\mathrm{T}$ as in Eq. (2) [17]. Force measure included mean absolute force (MF) in the horizontal direction which was normalized by body mass, M as in Eq. (3). Normalized GRF corresponds to the acceleration of whole body center of mass [12]. All measures were discomposed into anterioposterior and mediolateral directions. Test-retest reliability of all measures was acceptable (Cronbach's alpha was greater than 0.74 ). Average of three trials per subject was used further analysis.

$$
\begin{aligned}
& M D_{C O P}=\frac{\sum\left|C O P-C O P_{0}\right|}{N} \\
& M V_{C O P}=\frac{\sum|\Delta C O P|}{T} \\
& M F=\frac{1}{M} \cdot \frac{\sum|F|}{N}
\end{aligned}
$$

Two-way ANOVA was performed on the measures with the feet distance and gender as independent factors. Subsequently, post-hoc comparisons (repeated measure) were performed for the factors having significant main effect. Dependency among measures was evaluated by Pearson correlation.

Regarding the natural feet distance, reliability test and descriptive statistics were performed.

\section{Results}

Two-way ANOVA showed a significant effect of feet distance $(\mathrm{p}<0.001)$ but insignificant effect of gender or interaction (Table 1). Accordingly, gender was not differentiated in subsequent analysis. 
Table 1

Two-way ANOVA results

\begin{tabular}{lllll}
\hline \hline \multirow{2}{*}{$\begin{array}{l}\text { outcome } \\
\text { measures }\end{array}$} & \multirow{2}{*}{ direction } & Main effect & & \\
\cline { 3 - 5 } & & Feet distance & Gender & \\
\hline MD & anteroposterior & $* *$ & 0.38 & 0.40 \\
& mediolateral & $* *$ & 0.73 & 0.90 \\
\hline MV & anteroposterior & $* *$ & 0.85 & 0.10 \\
& mediolateral & $* *$ & 0.98 & 0.63 \\
\hline MF & anteroposterior & $* *$ & 0.25 & 0.46 \\
& mediolateral & $* *$ & 0.17 & 0.56 \\
\hline
\end{tabular}

Note: ${ }^{* *} \mathrm{p}<0.001$

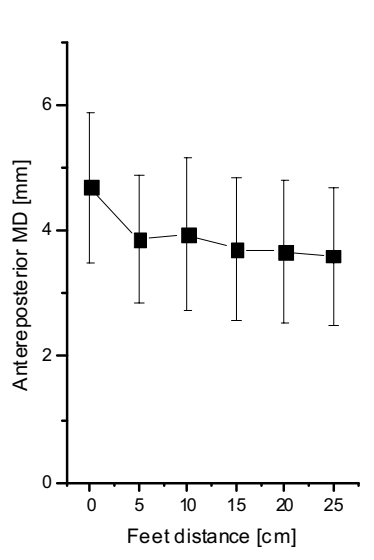

(a) mean distance of COP

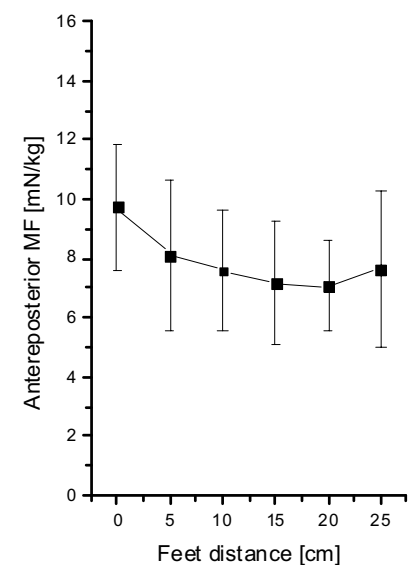

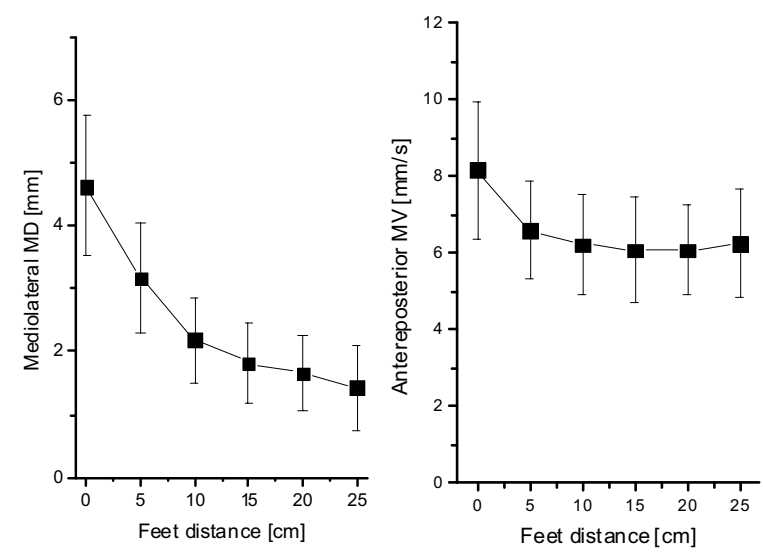

(b) mean velocity of COP
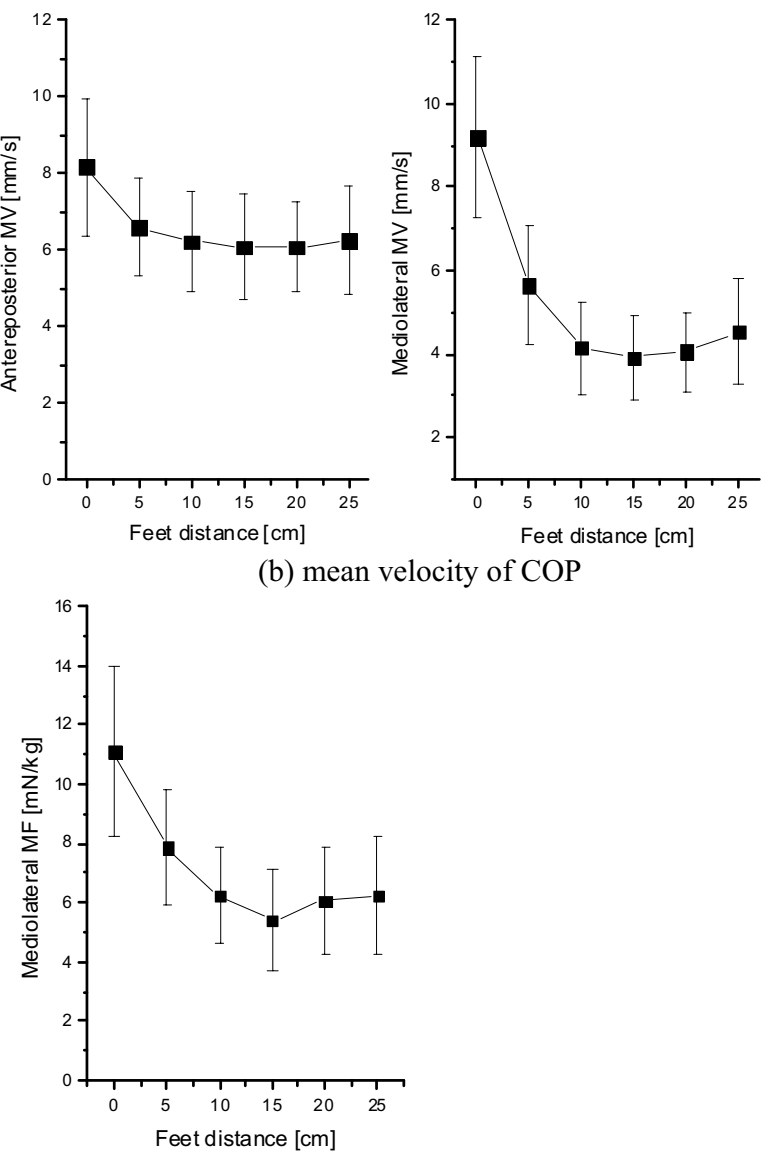

(c) mean horizontal GRF

Fig. 1. Effect of feet distance on outcome measures.

Figure 1 shows the effect of feet distance on outcome measures and Table 2 shows post-hoc comparisons (Tukey's HSD test). MD of COP showed monotonic decrease with feet distance in both anteroposterior and mediolateral directions, where the monotonicity was more evident for mediolateral direction. The smallest MD located at the greatest feet distance $(25 \mathrm{~cm})$ in both directions. 
Table 2

Post-hoc comparisons of outcome measures with feet distance as an independent factor

\begin{tabular}{|c|c|c|c|c|c|c|}
\hline measure & $\begin{array}{l}\text { feet distance } \\
{[\mathrm{cm}]}\end{array}$ & 5 & 10 & 15 & 20 & 25 \\
\hline \multirow{5}{*}{ MD anteroposterior } & 0 & $* *$ & $* *$ & $* *$ & $* *$ & $* *$ \\
\hline & 5 & & & & & \\
\hline & 10 & & & & & $*$ \\
\hline & 15 & & & & & \\
\hline & 20 & & & & & \\
\hline \multirow{5}{*}{$\begin{array}{l}\text { MD } \\
\text { mediolateral }\end{array}$} & 0 & $* *$ & $* *$ & $* *$ & $* *$ & $* *$ \\
\hline & 5 & & $* *$ & $* *$ & $* *$ & $* *$ \\
\hline & 10 & & & $* *$ & $* *$ & $* *$ \\
\hline & 15 & & & & & $*$ \\
\hline & 20 & & & & & $*$ \\
\hline \multirow{5}{*}{$\begin{array}{l}\text { MV } \\
\text { anteroposterior }\end{array}$} & 0 & $* *$ & $* *$ & $* *$ & $* *$ & $* *$ \\
\hline & 5 & & $*$ & $*$ & $* *$ & + \\
\hline & 10 & & & & & \\
\hline & 15 & & & & & \\
\hline & 20 & & & & & \\
\hline \multirow{5}{*}{$\begin{array}{l}\text { MV } \\
\text { mediolateral }\end{array}$} & 0 & $* *$ & $* *$ & $* *$ & $* *$ & $* *$ \\
\hline & 5 & & $* *$ & $* *$ & $* *$ & $* *$ \\
\hline & 10 & & & $*$ & & + \\
\hline & 15 & & & & & $* *$ \\
\hline & 20 & & & & & $* *$ \\
\hline \multirow{5}{*}{$\begin{array}{l}\text { MF } \\
\text { anteroposterior }\end{array}$} & 0 & $* *$ & $* *$ & $* *$ & $* *$ & $* *$ \\
\hline & 5 & & & + & $*$ & \\
\hline & 10 & & & & + & \\
\hline & 15 & & & & & \\
\hline & 20 & & & & & \\
\hline \multirow{5}{*}{ MF mediolateral } & 0 & $* *$ & $* *$ & $* *$ & $* *$ & $* *$ \\
\hline & 5 & & $* *$ & $* *$ & $* *$ & $* *$ \\
\hline & 10 & & & $*$ & & \\
\hline & 15 & & & & + & + \\
\hline & 20 & & & & & \\
\hline
\end{tabular}

Note: $+\mathrm{p}<0.05,{ }^{*} \mathrm{p}<0.01,{ }^{*} \mathrm{p}<0.001$

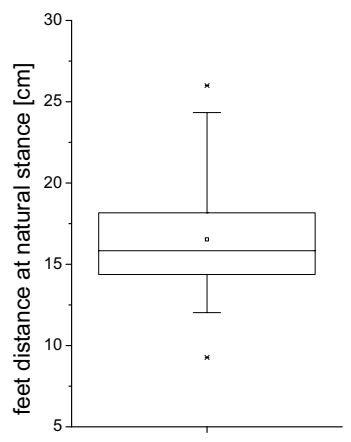

(c) mean horizontal GRF

Fig. 2. Feet distance at natural stance. 
Table 3

Correlations among outcome measures

\begin{tabular}{llllllll}
\hline \hline $\begin{array}{l}\text { outcome } \\
\text { measures }\end{array}$ & \multirow{2}{*}{ direction } & $\begin{array}{l}\text { MD } \\
\text { MP }\end{array}$ & ML & AP & ML & AP & ML \\
\hline MD & AP & & 0.51 & 0.42 & 0.48 & $\mathbf{0 . 7 1}$ & 0.57 \\
& ML & & & 0.49 & $\mathbf{0 . 8 7}$ & 0.58 & $\mathbf{0 . 7 5}$ \\
\hline MV & AP & & & & 0.53 & 0.56 & 0.50 \\
& ML & & & & & 0.60 & $\mathbf{0 . 8 0}$ \\
\hline MF & AP & & & & & & \\
& ML & & & & & & \\
\hline
\end{tabular}

Note: all correlation coefficients were significant $(\mathrm{p}<0.01)$.

In contrast, MV of COP showed U-shaped pattern, i.e., decrease followed by increase with respect to feet distance (Figure 1(b)). The smallest MV located at the feet distance of $20 \mathrm{~cm}$ (anteroposterior direction) and $15 \mathrm{~cm}$ (mediolateral direction). MF showed U-shaped pattern similar to but more evident than MV (Figure 1(c)). The smallest MF located at the feet distance of $20 \mathrm{~cm}$ (anteroposterior direction) and $15 \mathrm{~cm}$ (mediolateral direction), as was the case with $\mathrm{MV}$. The dimension of MF was $[\mathrm{mN} / \mathrm{Kg}]$ equivalent to $\left[\mathrm{mm} / \mathrm{s}^{2}\right]$ when it was converted to the acceleration of COM.

Test-retest reliability of natural feet distance investigated by Cronbach's alpha was 0.88 , which shows it is reproduction in each subject was reliable. Figure 2 shows a box plot of the feet distance at natural stance. Gender was not differentiated as there was no gender difference according to independent $t$-test $(p=0.59)$. The mean feet distance at natural stance was $16.5 \mathrm{~cm}(\mathrm{SD}: 3.8 \mathrm{~cm})$ with a $95 \%$ confidence interval of $15.3-17.7 \mathrm{~cm}$, which is comparable to those in the literature $[3,18]$.

Table 3 shows the Pearson correlation coefficients among measures. Though correlation coefficients for all pairs were significant, a high correlation ( $r>0.8)$ was shown only for mediolateral pairs (MD vs. MV and MV vs. MF). Moderate correlations ( $\mathrm{r}=0.7-0.8)$ were shown for MD and MF pairs in both anteroposterior and mediolateral directions.

\section{Discussion}

COP has been regarded to be an approximation of a center of mass (hence the resultant performance of postural control) from the inverted pendulum model when we assume the vertical GRF is predominant and the horizontal GRF can be ignored [11]. On the other hand, horizontal GRF is the direct cause of horizontal acceleration of COM of a whole body [12].

The results showed that the COP variations (MD in both directions) were the least for the widest feet distance, however, horizontal GRF (MF) had the minimum near the natural feet distance. Specifically, the average feet distance of natural stance $(16.5 \mathrm{~cm})$ located between the feet distance of the minimum mediolateral GRF $(15 \mathrm{~cm})$ and that of minimum anteroposterior GRF $(20 \mathrm{~cm})$, slightly closer to the former.

The results suggest that the effort of static postural control be minimized around the natural feet distance. Though the resultant postural sway (MD) was less at the wider stance, further reduction from that of natural stance $(15-20 \mathrm{~cm}$ ) was insignificant (Table 2$)$. Therefore, it is highly feasible that a human prefers the natural stance because the effort of postural control is minimized while maintaining an acceptable balance. In other words, a human may select a stance in terms of postural control effort. The present finding is new and may serve as a foundation for future researches of postural control. 
This strategy of a stance selection may reduce neuromuscular fatigue or may reserve neuromuscular control effort to cope with any unexpected disturbance, which should be further investigated. The mechanism why the natural stance lessens the postural control activity (MF) should also be elucidated in the future.

It is noted that MV of COP showed U-shape response similar to MF. Prieto et al. [16] speculated MV represent the amount of regulatory activity in postural corrections, but they failed to present the evidence. If their speculation was right, MV should be well correlated with MF, the direct cause of horizontal accelerations, in each direction. Table 3 shows that this was not true. That is, anteroposterior MV is little correlated with anteroposterior MF ( $\mathrm{r}=0.56)$. Mediolateral MV was highly correlated with mediolateral MF ( $\mathrm{r}=0.80)$, however, even higher correlation was shown with MD of the same direction $(\mathrm{r}=0.87)$. Therefore, MV seems to represent an aspect of postural control different from MF. The moderate correlation of MF with MD in both directions may represent the cause-result relationship of postural control.

\section{Acknowledgement}

This work was supported by Konkuk University.

\section{References}

[1] M. Piirtola and P. Era, Force platform measurements as predictors of falls among older people-a review, Gerontology 52 (2006), 1-16.

[2] J.W. Kim, G.M. Eom, C.S. Kim, D.H. Kim, J.H. Lee, B.K. Park and J. Hong, Sex differences in the postural sway characteristics of young and elderly subjects during quiet natural standing, Geriatr. Gerontol. Int. 10 (2010), 191-198.

[3] W.E. McIlroy and B.E. Maki, Preferred placement of the feet during quiet stance: Development of a standardized foot placement for balance testing, Clin. Biomech. 12 (1997), 66-70.

[4] J.T. Bingham, J.T. Choi and L.H. Ting, Stability in a frontal plane model of balance requires coupled changes to postural configuration and neural feedback control, J. Neurophysiol 106 (2011), 437-448.

[5] S. Kim, F.B. Horak, P. Carlson-Kuhta and S. Park, Postural feedback scaling deficits in Parkinson'S disease, J. Neurophysiol 102 (2009), 2910-2920.

[6] S.M. Henry, J. Fung and F.B. Horak, Effect of stance width on multidirectional postural responses, J. Neurophysiol 85 (2001), 559-570.

[7] A. Goodworth, A. Chandan, H. Chase, E. Foster, H. Francoeur, J. Michaud and K. Terry, Stance width influences frontal plane balance responses to centripetal accelerations, Gait Posture 37 (2013), 98-102.

[8] A.D. Goodworth and R.J. Peterka, Influence of stance width on frontal plane postural dynamics and coordination in human balance control, J. Neurophysiol 104 (2010), 1103-1118.

[9] R.L. Kirby, N.A. Price and D.A. MacLeod, The influence of foot position on standing balance, J. Biomech. 20 (1987), 423-427.

[10] B.L. Day, M.J. Steiger, P.D. Thompson and C.D. Marsden, Effect of vision and stance width on human body motion when standing: Implications for afferent control of lateral sway, J. Physiol. 469 (1993), 479-499.

[11] D.A. Winter, Human balance and posture control during standing and walking, Gait Posture 3 (1995), $193-214$.

[12] A. Karlsson and T. Persson, The ankle strategy for postural control-a comparison between a model-based and a markerbased method, Comput. Methods Programs Biomed. 52 (1997), 165-173.

[13] P.A. Goldie, T.M. Bach and O.M. Evans, Force platform measures for evaluating postural control: Reliability and validity, Arch. Phys. Med. Rehabil. 70 (1989), 510-517.

[14] E. Jonsson, A. Seiger and H. Hirschfeld, Postural steadiness and weight distribution during tandem stance in healthy young and elderly adults, Clin. Biomech. 20 (2005), 202-208.

[15] C. Bonnet, C. Carello and M.T. Turvey, Diabetes and postural stability: Review and hypotheses, J. Mot. Behav. 41 (2009), 172-190. 
[16] M. Schmid, S. Conforto, V. Camomilla, A. Cappozzo and T. D'Alessio, The sensitivity of posturographic parameters to acquisition settings, Med. Eng. Phys. 24 (2002), 623-631.

[17] T.E. Prieto, J.B. Myklebust, R.G. Hoffmann, E.G. Lovett and B.M. Myklebust, Measures of postural steadiness: Differences between healthy young and elderly adults, IEEE Trans. Biomed. Eng. 43 (1996), 956-966.

[18] J. Jang, K.T. Hsiao and E.T. Hsiao-Wecksler, Balance (perceived and actual) and preferred stance width during pregnancy, Clin. Biomech. 23 (2008), 468-476. 